\title{
Universiteit
}

Leiden

The Netherlands

\section{Defect-induced charge-order melting in thin films of Pr0.5Ca0.5MnO3}

Zhang, Y.Q.; Zhu, Y.; Zhang, Z.D.; Aarts, J.

\section{Citation}

Zhang, Y. Q., Zhu, Y., Zhang, Z. D., \& Aarts, J. (2007). Defect-induced charge-order melting in thin films of Pr0.5Ca0.5MnO3. Journal Of Applied Physics, 101, 063919.

doi:10.1063/1.2710341

Version: $\quad$ Not Applicable (or Unknown)

License: $\quad$ Leiden University Non-exclusive license

Downloaded from: https://hdl.handle.net/1887/45176

Note: To cite this publication please use the final published version (if applicable). 


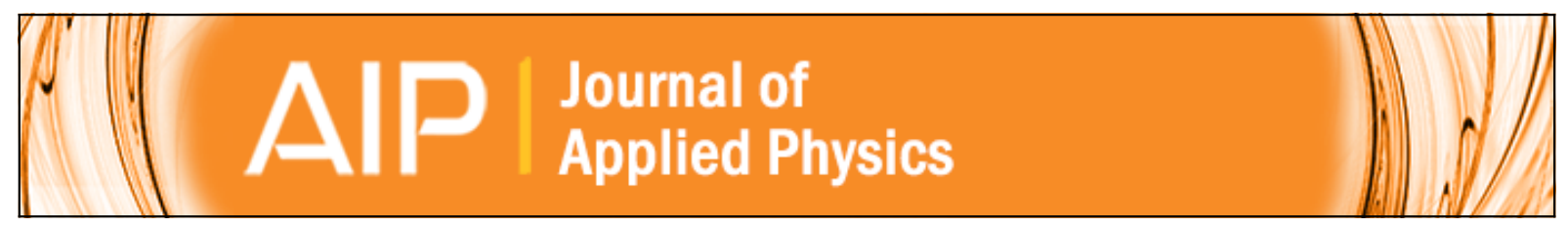

\section{Defect-induced charge-order melting in thin films of $\mathrm{Pr} 0.5 \mathrm{Ca} 0.5 \mathrm{Mn} \mathrm{O} 3$}

Y. Q. Zhang, Y. L. Zhu, Z. D. Zhang, and J. Aarts

Citation: Journal of Applied Physics 101, 063919 (2007); doi: 10.1063/1.2710341

View online: http://dx.doi.org/10.1063/1.2710341

View Table of Contents: http://scitation.aip.org/content/aip/journal/jap/101/6?ver=pdfcov

Published by the AIP Publishing

\section{Articles you may be interested in}

Evidence of substrate induced charge order quenching, insulator metal transition, and colossal magnetoresistance in polycrystalline $\operatorname{Pr} 0.58 \mathrm{Ca} 0.42 \mathrm{MnO} 3$ thin films

Appl. Phys. Lett. 96, 052512 (2010); 10.1063/1.3302455

Stress deformations and structural quenching in charge-ordered Sm $0.5 \mathrm{Ca} 0.5 \mathrm{MnO} 3$ thin films J. Appl. Phys. 98, 093903 (2005); 10.1063/1.2126157

Magnetotransport properties in thin films of charge-ordered materials

J. Appl. Phys. 89, 8057 (2001); 10.1063/1.1372369

Strain-stabilized charge ordering and magnetorelaxor behaviors in Cr-doped $\operatorname{Pr} 0.5 \mathrm{Ca} 0.5 \mathrm{MnO} 3$ epitaxial thin films

Appl. Phys. Lett. 78, 3505 (2001); 10.1063/1.1376146

Spectacular decrease of the melting magnetic field in the charge-ordered state of $\operatorname{Pr} 0.5 \mathrm{Ca} 0.5 \mathrm{MnO} 3$ films under tensile strain

Appl. Phys. Lett. 77, 1023 (2000); 10.1063/1.1288673

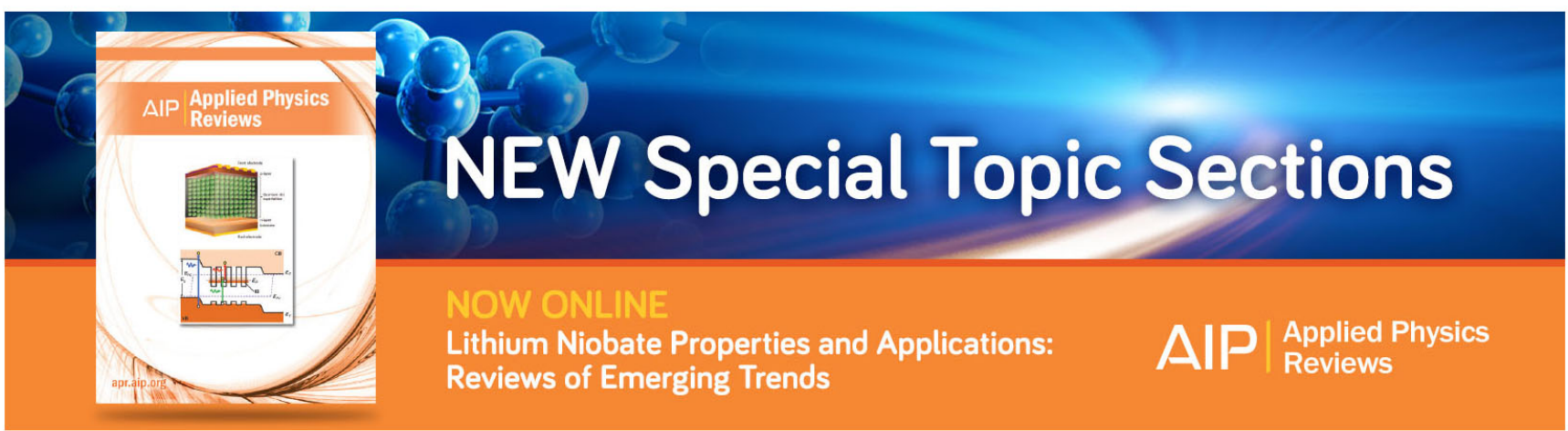




\title{
Defect-induced charge-order melting in thin films of $\operatorname{Pr}_{0.5} \mathrm{Ca}_{0.5} \mathrm{MnO}_{3}$
}

\author{
Y. Q. Zhang, ${ }^{\text {a) }}$ Y. L. Zhu, and Z. D. Zhang \\ Shenyang National Laboratory for Materials Science, Institute of Metal Research, Chinese Academy \\ of Science, 72 Wenhua Road, Shenyang 110016, People's Republic of China and International Center \\ for Materials Physics, Chinese Academy of Science, 72 Wenhua Road, Shenyang 110016, \\ People's Republic of China \\ J. Aarts \\ Kamerlingh Onnes Laboratory, Leiden University, P.O. Box 9504, 2300 RA Leiden, The Netherlands
}

(Received 15 December 2006; accepted 10 January 2007; published online 30 March 2007)

\begin{abstract}
We have investigated the relation between defect structure and charge order melting in thin films of epitaxial $\mathrm{Pr}_{0.5} \mathrm{Ca}_{0.5} \mathrm{MnO}_{3}$ (PCMO), grown under strain on $\mathrm{SrTiO}_{3}$. We compared the behavior of an $80 \mathrm{~nm}$ film grown in one deposition step at $840{ }^{\circ} \mathrm{C}$ with the behavior of a film grown in two steps. In the two-step case, a thin PCMO layer of $10 \mathrm{~nm}$ was deposited at $120^{\circ} \mathrm{C}$, followed by $70 \mathrm{~nm}$ deposited at $840{ }^{\circ} \mathrm{C}$. The increase of the growth temperature leads to complete crystallization of the first layer and the lattice constants of the two-step grown film indicate that tensile strain is still present. On the other hand, a magnetic field of only $5 \mathrm{~T}$ is required to melt the charge-order state in the two-step grown film, which is a much lower than the value for the normally grown film. This appears to be connected to a larger amount of threading dislocations present in the first (recrystallized) layer. (C) 2007 American Institute of Physics. [DOI: 10.1063/1.2710341]
\end{abstract}

\section{INTRODUCTION}

One of the attractive features of thin films of colossal magnetoresistance (CMR) perovskite manganese oxide films is that, to a certain degree, the properties can be varied by playing with strain to be controlled by using different substrates with lattice constants which do not match those of the bulk material. In this way, it was, for instance, shown that the ferromagnetic transition temperature in $(\mathrm{La}, \mathrm{Ba}) \mathrm{MnO}_{3}$ can be increased $^{1}$ or that different types of spin and orbital orders can be engineered in ( $\mathrm{La}, \mathrm{Sr}) \mathrm{MnO}_{3}{ }^{2}{ }^{2}$ Strain can also influence the onset of charge ordering. In bulk $\mathrm{Pr}_{0.5} \mathrm{Ca}_{0.5} \mathrm{MnO}_{3}$ (PCMO), with a $1: 1$ ratio of $\mathrm{Mn}^{3+}: \mathrm{Mn}^{4+}$, charge order and orbital order (called the COO transition) simultaneously set in around $T_{\mathrm{COO}}=250 \mathrm{~K} .{ }^{3}$ Upon further cooling, an antiferromagnetic (AF) state forms at $170 \mathrm{~K}^{4}$ By applying a magnetic field, the combined $\mathrm{AF} / \mathrm{COO}$ state can go through a first-order phase transition (called the melting of the charge order) and transform to a ferromagnetic metallic state. The melting fields are quite high, around $27 \mathrm{~T}$ at low temperatures. ${ }^{5}$ When thin films are grown on a substrate with a larger lattice constant such as $\mathrm{SrTiO}_{3}$ (STO), the strain stabilizes the $\mathrm{COO}$ state and can significantly increase $T_{\mathrm{COO}}$; for thin films (thickness less than $25 \mathrm{~nm}$ ), values of $T_{\mathrm{COO}}$ above $320 \mathrm{~K}$ were reported, ${ }^{6}$ much higher than that of the bulk. Melting fields, however, are always lower than the bulk values, which was ascribed either to the effect of substrate clamping ${ }^{7}$ or to the effects of disorder induced by strain relaxation. ${ }^{8}$ The latter mechanism would explain both the thickness dependence of the melting behavior (with thicker films showing lower melting fields) and the effect of postannealing, which relaxes strain, increases disorder, and lowers the melting fields.

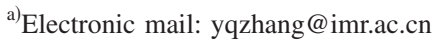

Recently, it was reported ${ }^{9,10}$ that strain relaxation of epitaxial STO films grown on $\mathrm{LaAlO}_{3}$ can be accelerated effectively by a two-step growth technique. In this paper, we investigate what the effect of such deposition is on the structure and the charge ordered melting behavior of a typical PCMO film of $80 \mathrm{~nm}$.

\section{EXPERIMENTAL DETAILS}

The PCMO films were deposited on $\mathrm{SrTiO}_{3}$ substrates by sputtering in pure oxygen atmosphere of $300 \mathrm{~Pa}$ with substrate-source on-axis geometry. A two-step grown film of $80 \mathrm{~nm}$, to be compared to an $80 \mathrm{~nm}$ film grown earlier in normal fashion, was prepared by the following procedure: a thin layer of $10 \mathrm{~nm}$ was first grown at $120^{\circ} \mathrm{C}$; after deposition of this layer, the temperature of the substrate was increased to $840^{\circ} \mathrm{C}$ and kept constant for $30 \mathrm{~min}$; then the second layer of $70 \mathrm{~nm}$ was grown at this temperature. The crystal structure and lattice constants were investigated by means of x-ray diffraction and high-resolution electron microscopy (HREM). The out-of-plane lattice parameter was determined from the $(001)_{c},(002)_{c}$, and $(003)_{c}$ reflections $(c$ refers to the pseudocubic unit cell, with the $b$ axis taken perpendicular to substrate). The in-plane lattice parameters were determined from the $(103)_{c}$ and $(203)_{c}$ reflections. The magnetotransport properties of the films were measured with a physical properties measurement system (PPMS) at temperatures between 300 and $10 \mathrm{~K}$ in magnetic fields up to $9 \mathrm{~T}$.

\section{RESULTS AND DISCUSSION}

Turning first to the x-ray data, bulk PCMO crystallizes in orthorhombic Pnma structure with $a=0.5395 \mathrm{~nm}, b$ $=0.7612 \mathrm{~nm}$, and $c=0.5403 \mathrm{~nm}$. In terms of a pseudocubic lattice parameter $a_{c}$, there is a slight difference between the $a-c$ plane $\left(a_{c}=0.3818 \mathrm{~nm}\right.$, called $\left.a_{\text {in }}\right)$ and the $b$ axis $\left(a_{c}\right.$ 


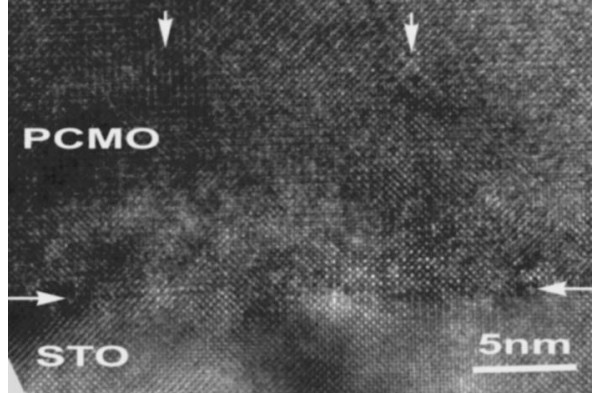

FIG. 1. High-resolution TEM image of two-step grown PCMO film of $80 \mathrm{~nm}$.

$=0.3806 \mathrm{~nm}$, called $\left.a_{\text {out }}\right)$. For a single film of $7 \mathrm{~nm}$ deposited at $120{ }^{\circ} \mathrm{C}$, no reflections of the film could be found in $\mathrm{x}$-ray diffraction, confirming the more or less amorphous state of such a layer, before the next layer is deposited. For the twostep grown film, we find $a_{\text {in }}=0.38376 \mathrm{~nm}$ and $a_{\text {out }}$ $=0.37869 \mathrm{~nm}$, which can be compared to the earlier result for a normally grown film of $a_{\text {in }}=0.38421 \mathrm{~nm}$ and $a_{\text {out }}$ $=0.37961 \mathrm{~nm} .{ }^{8}$ Comparing these values to the bulk values, it is evident that the two-step grown film also is under tensile strain, only somewhat less than the normally grown film. Moreover, the full width at half maximum of the rocking curve of all diffraction peaks is less than $0.3^{\circ}$, indicating good crystallinity for this film. This is confirmed by HREM data, as shown in Fig. 1. The picture shows that the first thin layer of $10 \mathrm{~nm}$ has crystallized completely after annealing. The first conclusion therefore is that, even after starting the growth on the amorphous layer, epitaxial conditions are recovered in the postannealing and the subsequent growth, including the strained state. This is different from the findings in Refs. 9 and 10, where almost full relaxation was achieved. Although the reason for this would need further investigation, we note that the deposition technique in that case (laser ablation) is different from our sputtering method.

Next we measured the magnetotransport properties of the two-step grown film. Figure 2 shows resistance $R$ versus temperature $T$, which can be used to determine the chargeordering temperature $T_{\mathrm{COO}}$. In bulk material this is directly

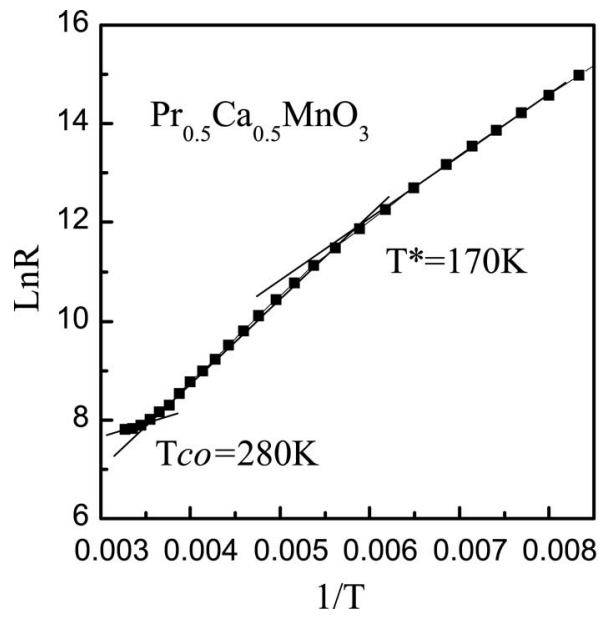

FIG. 2. Charge order temperature and its transition width of two-step grown PCMO film.
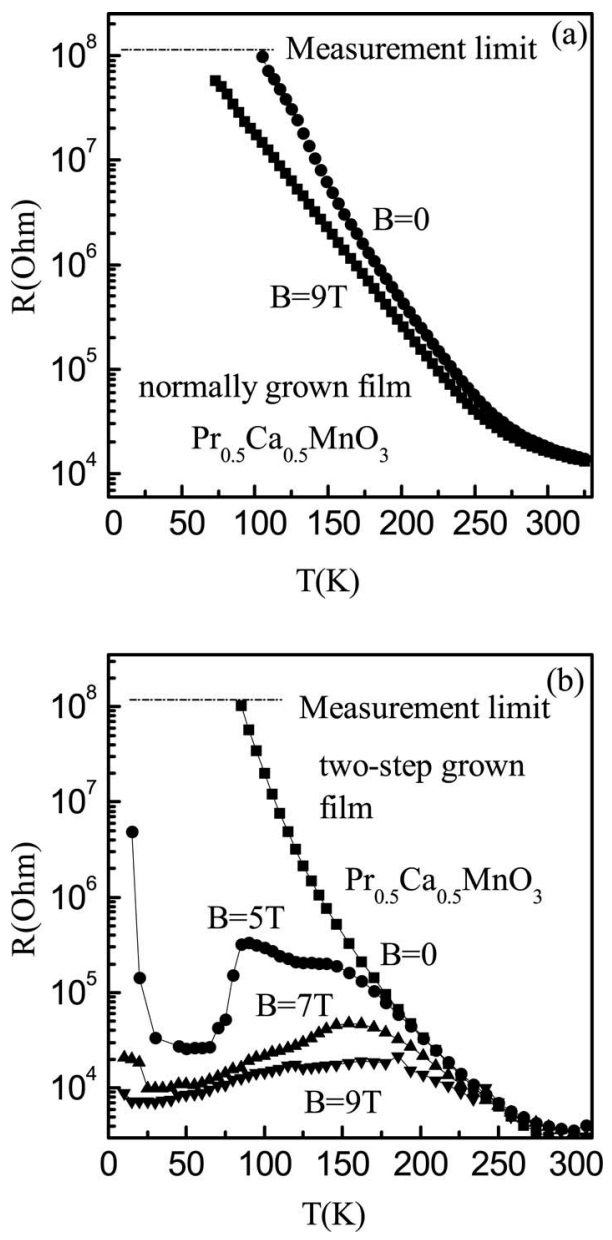

FIG. 3. Temperature dependence of resistance at different magnetic fields for (a) the normally grown film and (b) the two-step grown film.

visible in an upward step of the resistance when lowering the temperature through $T_{\mathrm{COO}}$. In films, this signature is both weaker and less sharply defined, but we showed before that $T_{\mathrm{COO}}$ can be extracted by plotting $\ln (R / 1 \Omega)$ against $1 / T^{8}{ }^{8}$ This is also done in Fig. 2. For the two-step grown film we find $T_{\mathrm{COO}}=280 \mathrm{~K}$, very close to the value of $285 \mathrm{~K}$ for the normally grown film. On the other hand, the transition width $\Delta T_{\text {tr }}$ for the former is $110 \mathrm{~K}$, significantly larger than the $75 \mathrm{~K}$ found for the latter. It indicates a larger amount of disorder present in the two-step grown film, as can also be seen by comparing this result to the earlier one of a normally grown film of $50 \mathrm{~nm}$ (Ref. 11) $\left(\Delta T_{\mathrm{tr}}=64 \mathrm{~K}\right.$, similar to the $80 \mathrm{~nm}$ film) versus a postannealed film of $50 \mathrm{~nm}\left(\Delta T_{\text {tr }}\right.$ $=124 \mathrm{~K})$. The postannealing treatment in that case also significantly lowered the melting field. Figures 3(a) and 3(b) show $R(T)$ for the normally grown film and the two-step grown film in different magnetic fields of $0,5,7$, and $9 \mathrm{~T}$ applied parallel to the film plane and to the direction of current flow. The normally grown film shows insulating behavior in the whole temperature range up to $9 \mathrm{~T}$ (the maximum field in our PPMS). For the two-step grown film, the charge order state melts in a magnetic field of $5 \mathrm{~T}$. So, both $\Delta T_{\mathrm{tr}}$ and the melting behavior reveal a significantly weakened charge order state in the two-step grown film. To understand this better, we return to the film morphology.

A low-magnification bright field image of the two-step 


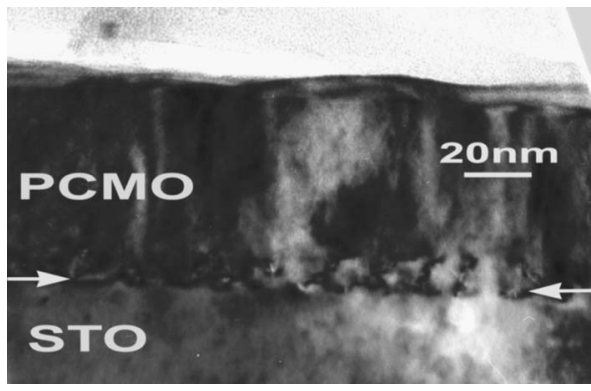

FIG. 4. Low-magnification bright field image of two-step grown PCMO film.

grown PCMO film is shown in Fig. 4. Many threading dislocations exist in the first layer of $10 \mathrm{~nm}$, as marked with two white arrowheads, which strongly indicates that both the increased disorder and the somewhat larger strain relaxation of the two-step grown PCMO film are due to the increased amount of misfit dislocations in the first layer, as compared to the situation in a normally grown film. Still, the scenario sketched in Refs. 9 and 10 appears to work less well in our case. There, the annealing of the first layer led to strong relaxation which was explained by arguing that existing as well as newly created misfit dislocations move to the interface. In our case, some relaxation can be observed, but more important for the physical properties of the system is the larger disorder which is induced in the second layer, leading to a substantial reduction of the charge order melting field of the two-step grown film, as compared to the normally grown film.

\section{CONCLUSIONS}

In summary, we have investigated the growth of a PCMO film of $80 \mathrm{~nm}$ using the two-step growth technique. A magnetic field of only $5 \mathrm{~T}$ is required to collapse (melt) the charge-ordered state in this film, which is substantially lower than one needed in the normally grown film (above $9 \mathrm{~T}$ ). The bright field image of the two-step grown film shows that more threading dislocations in the first layer are produced before the deposition of the second layer, which results in more relaxation of the in-plane tensile strain of the two-step grown film, but also to more disorder in the second layer, which is the cause for the reduction of the melting fields.

\section{ACKNOWLEDGMENTS}

This work has been carried out in collaborative research project (No. 04CDP020) under the auspices of the Dutch Royal Academy of Sciences (KNAW) and the Chinese Academy of Sciences, and was also supported by National Natural Science Foundation of China under Grant No. 50332020 and 50171070.

${ }^{1}$ Y. Lu et al., Phys. Rev. B 62, 15806 (2000).

${ }^{2}$ Y. Tokura and N. Nagaosa, Science 288, 462 (2000).

${ }^{3}$ R. Kajimoto, H. Yoshizawa, Y. Tomioka, and Y. Tokura, Phys. Rev. B 63, 212407 (2001).

${ }^{4}$ Z. Jirák, F. Damay, M. Hervieu, C. Martin, B. Raveau, G. André, and F. Bourée, Phys. Rev. B 61, 1181 (2000).

${ }^{5}$ M. Tokunaga, N. Miura, Y. Tomioka, and Y. Tokura, Phys. Rev. B 57, 5259 (1998).

${ }^{6}$ Z. Q. Yang, Y. Q. Zhang, J. Aarts, M. Y. Wu, and H. W. Zandbergen, Appl. Phys. Lett. 88, 072507 (2006).

${ }^{7}$ W. Prellier, A. M. Haghiri-Gosnet, B. Mercey, Ph. Lecoeur, M. Hervieu, Ch. Simon, and B. Raveau, Appl. Phys. Lett. 77, 1023 (2000).

${ }^{8}$ Z. Q. Yang, R. W. A. Hendrikx, P. J. M. van Bentum, and J. Aarts, Europhys. Lett. 58, 864 (2002).

${ }^{9}$ T. Yamada, K. F. Astafiev, V. O. Sherman, A. K. Tagantsev, S. Dong, P. Muralt, and N. Setter, J. Appl. Phys. 98, 054105 (2005).

${ }^{10}$ T. Yamada, K. F. Astafiev, V. O. Sherman, A. K. Tagantsev, P. Muralt, and N. Setter, Appl. Phys. Lett. 86, 142904 (2005).

${ }^{11}$ Z. Q. Yang, Ph.D. thesis, Leiden University, 2004. 